\title{
Der Islam als Problem.
}

\section{Von}

\author{
․ C. H. Becker.
}

Die Wissenschaft vom Islam, der in dieser Zeitschrift ein neues Heim eröffnet werden soll, braucht ihre Existenzberechtigung nicht erst zu erweisen. Wie die alte Geschichte neben die klassische Philologie ist sie seit langem als historische Disziplin neben die Philologien der von Muhammedanern gesprochenen Sprachen getreten.

Auch wäre es eine allzubequeme Aufgabe, in diesem einleitenden Aufsatz die zahlreichen Probleme zu skizzieren, deren Lösung wir in dieser Zeitschrift fördern wollen. Wer möchte sich vermessen, hier die Wege weisen zu wollen, ohne gleich selber ernstlich mitzuarbeiten?! Nein, nicht von $\mathrm{d}$ e $\mathrm{n}$ Problemen des Islam, sondern von $\mathrm{d}$ e $\mathrm{m} \mathrm{I} \mathrm{s} \mathrm{l}$ a m als Problem soll hier die Rede sein.

Wir alle brauchen das Wort Islam in einer Fülle von Bedeutungen. Wir verstehen darunter zunächst die Relig i on und zwar sowohl die primitive Predigt Muhammed's, als auch das davon grundverschiedene orthodoxe Lehrgebäude oder die Volksreligion der heutigen Muhammedaner in Asien und Afrika. Ob wir die religiöse Betätigung der Türken oder der Neger meinen, ob wir von einem Gazālī sprechen oder von einem sudanesischen Mahdī - wir reden schlechthin vom Islam. Je weniger die Leute davon wissen, desto mehr verallgemeinern sie. Wer würde dagegen wagen, ohne sich lächerlich zu machen, abessinische Kirchenzustände kurz als Christentum zu bezeichnen? Oder gar sie ohne weiteres mit dem protestantischen Christentum in einem Atem zu nennen?

Damit nicht genug, bezeichnen wir mit Islam eines der großen orientalischen $W e l t r e i c h e$, zahllose Einzelstaaten, dic auf seinen Trümmern erwuchsen, ja sogar die muhammedanischen Staaten der Gegenwart. Aber nicht nur wirkliche Staaten, ein viel GröBeres nennen wir so: eine politis che Theorie, mag sie sich nun auf staatsrechtlicher oder eschatologischer Lehre aufbauen.

Islam. I. 
Und endlich nennen wir das Religion und Staat umschlingende Kulturganze mit dem gleichen Namen: eine $Z$ i vilis a ti o $n$, die bei aller lokaler Verschiedenheit, bei weiten zeitlichen Abständen doch scheinbar ein einheitliches Gepräge trägt. So einheitlich, so charakteristisch und ausschlaggebend scheint uns die Zugehörigkeit zur Religion, zum Staat, zur Zivilisation des Islam, daß die große Menge unter Islam selbst Völker von so heterogener Rassenbeschaffenheit wie Arier, Semiten und Neger wie unter einem Sammelnamen begreift.

Je tiefer wir eindringen, desto mehr werden wir differenzieren, und man kann nicht genug dazu raten, namentlich bei vergleichenden Werturteilen immer recht genau zu umschreiben, was man im einzelnen Falle unter Islam versteht. Aber bei aller Vorsicht und Schärfe in der begrifflichen Scheidung wird doch auch der Spezialist immer wieder den Sammelnamen Islam schlechthin anwenden. Ist das berechtigt? D. h. finden sich alle die geschilderten Einzelzweige tatsächlich widerspruchslos unter dem Oberbegriff Islam zusammen, der doch zunächst und hauptsächlich eine Religion bezeichnet? Dadurch, daß wir diesen Namen auf den Titel unserer Zeitschrift setzten, haben wir von vornherein Stellung $\mathrm{zu}$ dieser Frage genommen.

Bei der Zergliederung des Wortes Islam vergegenwärtigten wir uns bereits die Hauptfaktoren, die in ihrem Zusammenwirken den als Einheit empfundenen Begriff Islam erzeugen: Das einheitliche Bekenntnis, das einheitliche politische Ideal und die bei aller lokalen Differenzierung wenigstens in den Idealen zum. Teil auch in der Praxis einheitliche Zivilisation. Daß diese Faktoren durch die Religion verbunden sind, daß Staatsidee und Zivilisation nur durch ihre religiöse Begründung Geltung haben, steht außer Zweifel. In der Gegenwart wenigstens ist die islamische Religion ein starkes einigendes Band, das die zentrifugalen nationalen Kräfte der Völker zu einem großen Ganzen zusammenschließt. Wenn der Mngindomann in DeutschOstafrika den Islam annimmt, nennt er als seinen Stamm hinfort nicht mehr die Wangindo, sondern den Islam ${ }^{\mathrm{x}}$ ). Der Araber verbrüdert sich mit dem bekehrten Neger, und in dem religiösen Zentrum Mekka laufen alle diese Fäden zusammen. Namentlich dem christlichen Europa gegenüber fühlen sich die Muhammedaner der ganzen Welt als eine Einheit. Da nun die Bedeutung der Religion eine mehr oder minder ähnliche Gestaltung des täglichen Lebens bedingt, so dürfen wir getrost von einer is 1 a $m$ is $c h$ en $E$ in he its $z$ i vili -

1) Missions-Blätter (Organ der St. Benediktus-Missions-Genossenschaft zu St. Ottilien) XIII, Heft 9 S. I3o. 
s a t i o n reden, wobei wir uns bewußt sind, daß die Religion in ihr den Ausschlag gibt.

Diese für die Gegenwart unbestreitbare Tatsache hat das Verständnis des geschichtlichen Werdegangs des Islam ganz wesentlich erschwert. Da in der Gegenwart die Religion als allbestimmendes Moment lebt und wirkt, da ferner die ganze historische Erscheinung des Islam auf einen Religionsstifter zurückging, was war da natürlicher als die Religion für den Faktor zu halten, dem das Verdienst, die islamische Einheitszivilisation geschaffen $z u$ haben, hauptsächlich, wenn nicht ausschließlich zukam?!

Dazu kam die uns vom Mittelalter überkommene klerikale An. schauungsweise, wie sie dem Islam gegenüber noch heute als communis opinio gilt. Das-Mittelalter wie die werdende Gegenwart sahen im Islam in erster Linie die feindliche Religion, die der Ausbreitung des Christentums einen Damm entgegensetzte, ja selbst seinen Besitzstand bedrohte. Die neue Religion, so legte man sich die historische Entwicklung zurecht, begeisterte die Araber, das Bedürfnis der Weltbekehrung trieb die Muslime hinaus. Mit dem Schwert verbreiteten sie ihre Religion. Muhammed war Prophet und Staatsmann in einer Person; damit war der Weltreichgedanke gegeben. In diesem neuen Staate schuf dann die arabische Kultur und die neue islamische Religion die arabisch-islamische Zivilisation. Wenn auch eine große Menge vorislamischer Ideen und Institutionen bestehen blieb, die Religion war nicht nur das primum movens, sie war der Neubildner, der Organisator einer ganzen Zivilisation. Die ganze spätere Entwicklung vollzog sich dann als Folg e der religiösen Gründung. So war es nur natürlich, daß die $\mathrm{R}$ e 1 i g i o n dieser ganzen Zivilisation einen einheitlichen Stempel aufdrückte. Damit war der Begriff einer islamischen Einheitszivilis a tion gegeben.

Trat man mit diesen an einer falschen Vorstellung von der christlichen Entwicklung ${ }^{2}$ ) großgezogenen Ideen an die arabischen Autoren heran, so fand man sie vollauf bestätigt; denn auch die islamischen Quellen sind durchaus klerikal orientiert, auch bei ihnen ist die ganze islamische Entwicklung die Schöpfung Muhammed's und der ersten goldenen Zeit der orthodoxen Kalifen. Staat und Gesellschaft, Wissenschaft und Wirtschaftsleben stehen unter der Herrschaft der religiösen Formel. Wuchert im Ziergarten der Theorie hie und da ein wildes

3) Ich denke besonders an ERsst TRÖrsen, Die'Soziallehren der christlichen Kirchen (Archiv für Sozialwissenschaft Bd. XXVI ff.). Auch bekenne ich hier dankbar, daB ich in der gesamten Fragestellung dieses Aufsatzes stark von Trö ztscu'schen hier und anderweitig niedergelegten Gedanken beeinfluBt bin. 
Reis empor, so wird es als mißbräuchliche Praxis hinweggewiesen. Die Welt des Islam ist beherrscht von der Religion in Vergangenheit und Gegenwart - wenigstens in der Theorie.

Erst in den letzten Jahrzehnten, jedenfalls erst lange nach ALFRED VON KREMER'S so verdienstlicher Kullurgeschichte, setzte die moderne Kritik ein. Allmăhlich rang sich die Forschung aus dem Bann der islamischen Tradition; wir lernten in Politik und Recht, in Religion und Leben, Theorie und Praxis unterscheiden. Wir sahen im Kampfe zwischen religiöser Forderung und Volkssitte die letztere siegen, wir erkannten im Ringen der Ideen die religiöse Färbung in unzähligen Fällen als reine literarische Form. Wir sahen das religiöse Recht sich entwickeln nicht an der Praxis, sondern im Gegensatz $z u$ ihr, wir überzeugten uns schließlich, daß auch die Gründer des arabischen Weltreiches gar nicht ihre Religion propagierten, sondern nur die weltliche Herrschaft der Araber. Gibt uns das alles nicht zu denken? Sind gegenüber diesen Tatsachen unsere herkömmlichen Anschauungen von der Rolle, die die Religion im Islam als Konstituante spielt, nicht außerordentlich $\mathrm{r}$ ef or mbedürftig ?

Das Problem ist eben unendlich viel komplizierter, als es sich dem ersten Blick darstellt. Kein Mensch wird bezweifeln wollen, daß der Islam seine Entstehung einem religiösen Erlebnis verdankt. Aber zwischen der religiösen Grundstimmung in der Seele Muhammed's und der panislamischen Tendenz der Gegenwart liegt eine g r o B e K $1 \mathrm{u} \mathrm{ft}$, die der Laie stets ohne weiteres überspringt. Wie wird die religiöse Idee des Stifters über alle Widerstände Herr, oder wie entwickelt sich aus dem religiösen Gedanken des Individuums die weltumspannende Einheitszivilisation des Islam, in der dann tatsächlich die Religion den Ausschlag gibt? Wenn wir uns zu dieser Untersuchung frei machen von der überkommenen Anschauungsweise, so werden wir wohl die große Bedeutung der Religion anerkennen, gleichzeitig aber auch konstatieren müssen, daß ihr doch noch in viel höherem Maße die Früchte eines ungeheuren politischen Machtwillens und die Resultate einer unabänderlichen wirtschaftlichen Entwicklung in den Schoß fallen. Die Religion blüht und gedeiht auf dem Boden, den andere Mächte gedüngt und vorbereitet haben. Das Problem des Islam ist also die Frage: Wie kommt die Einheitszivilisation des Islam zustande und welche Rolle spielt das religiöse Moment in diesemEntwicklungsprozeB?

Wir beginnen mit einer Analyse der Ausbreitung des Islam als Religion. Wenn irgendwo, so sollte man voraussetzen, daß die Ausbreitung der Religion ausschließlich oder doch 
hauptsächlich der religiösen Energie, dem Bedürfnis des Proselyten. machens zu danken ist.

In der mekkanischen Zeit ist das auch zweifellos der Fall. Ich kann dem Historiker nicht zustimmen, der in Muhammed von Anfang an den Politiker sieht, der je nach der politischen Opportunität zwischen Christentum und Heidentum hin und her schwankt $x$ ). Der Politiker erwacht in Muhammed erst mit dem Erfolg. Ein Ausharren und Entsagen, wie es Muhammed in Mekka durchzumachen hatte, kann nicht auf politischen Ehrgeiz, sondern nur auf eine tiefe innere religiöse Gewißheit zurückgehen. In den alten Suren des Qorān's spricht ein nicht gerade phantasiereicher, aber ein ehrlicher Schwärmer und kein intellektueller Energiemensch. Aber jeder, der den Orientalen und besonders den Araber kennt, weiß, wie widerspruchslos Frömmigkeit und Eigennutz, transzendentale Spekulation und weltliche Profitlichkeit in seiner Seele beieinander wohnen. Muhammed hat die Mekkaner an seinem religiösen Erlebnis teilnehmen lassen wollen, die Idee eines islamischen Weltreiches ist ihm, wenn überhaupt, erst in seinen letzten Lebensjahren gekommen. Wir, wie unsere Quellen, beurteilen diese Dinge stets zu sehr auf Grund unserer Kenntnis des tatsächlichen späteren Geschehens. Die Anfänge des Islam sind also $r$ ei n religi ös.

Mit dem Moment der Übersiedelung nach Medina tritt für Muhammed und seine Genossen unbewußt, um so greifbarer aber für den Kritiker der Motive ihres Handelns, der polit is che Macht$\mathrm{g} \mathrm{edanke} \mathrm{als} \mathrm{Stimulans} \mathrm{neben} \mathrm{die} \mathrm{Religion.} \mathrm{Von} \mathrm{da} \mathrm{ab} \mathrm{ist} \mathrm{die} \mathrm{Parole}$ nicht mehr Bekehrung zur Religion Allāh's, sondern Unterwerfung unter seinen Propheten. Mag schon bei den Individuen der nächsten Umgebung die Annahme des Islam nur zum geringsten Teil aus religiösem Bedürfnis erfolgt sein, die stammweisen Übertritte sind durchweg politischer Art. So sehen wir die Ausbreitung der islamischen Religion aufs engste verbunden mit der Vorherrschaft Medinas, und noch ehe der Islam die Grenzen Arabiens überschritt, ist aus einer religiösen Bewegung eine im wesentlichen politische geworden. Aber • so- lange der Islam noch auf Arabien beschränkt ist, nehmen die Angehörigen des neuen Staates, wenigstens in der Mehrheit, noch seine Religion an, so äußerlich und formal das auch gewesen sein mag.

Ganz anders wird die Sachlage in dem Augenblick, da der junge Staat die alten Kulturreiche Vorderasiens und Nordafrikas erobert. $\mathrm{Da}$ trennt sich scharf und klar die Ausbreitung des Islam als Staat

1) Huco Wisckuer, Arabisch-Semitisch-Orienlalisch, MVAG 1901 (4) S. 52 ff. 
von der der Religion; denn das alte Ammenmärchen, daß die Araber dem vorderen Orient ihre Religion mit dem Schwerte aufgezwungen hătten - jenes Hauptrüstzeug einer bequemen christlichen Polemik braucht an diesem Orte wohl nicht ausführlich widerlegt zu werden. Die Unterworfenen behielten uberall freie Religionsübung, sofern sie sich politisch unter die Oberhoheit des arabisch-islamischen Staates stellten. Diese Tatsache zeigt besser als alles andere, daß der Islam nicht mehr die auf Propaganda des Glaubens bedachte Religion des mekkanischen Muhammed war; sie mag ein Kompromiß mit der politischen Möglichkeit gewesen sein, sie ist aber hauptsächlich der Ausdruck dafür, daß das national arabische Moment das religiös-universelle überwog. Von Ausnahmen abgesehen, dachten die Araber zunächst gar nicht daran, die Unterworfenen zu b e k e h r e n. Sie wollten sich nach Art der modernen Kolonisatoren als Oberschicht über der Masse der zahlenden Heloten halten. Bürger des Staates war nur der Araber, der nach Art einer modernen Staatsangehörigkeit den Charakter eines Muslim erwarb. Die polit is che Eroberungdesvor. deren Orients durch die Araber ist also sachlich wiezeitlich von seinerIslamisierung scharf $z$ u trennen. Dazwischen liegen ein bis zwei, ja drei Jahrhunderte. Zur Lösung unserer Aufgabe bedarf es also einer getrennten Untersuchung dieser beiden für die Entwicklung des Islam zur Einheitszivilisation ausschlaggebenden historischen Prozesse.

Die Ausbreitung des Islam als $\mathrm{S} t \mathrm{a}$ a $\mathrm{t}$ kann nach dem Gesagten keinenfalls hauptsächlich auf das Konto des religiösen Enthusiasmus gesetzt werden. Der erlösende Gedanke, der uns die ganze Neugestaltung des Orients im 7. Jahrhundert erklärt, ist der Begriff der V ö $1 \mathrm{k} \mathrm{e} \mathrm{r-}$ wa'n d e rung. Man hat wohl schon immer die Siegeszüge der Araber a u $\mathrm{ch}$ als Völkerwanderung angesehen, man hatte auch wirtschaftliche Gründe zu ihrer Erklärung angeführt, aber es überwog doch bis vor kurzem überall der religiöse Gedanke. Es ist das unleugbare Verdienst Hugo WinckLER'S ${ }^{\mathrm{I}}$ ), die Tatsache der arabischen Völker-

- wanderung in ihrer ganzen historischen Tragweite erkannt zu haben. Seine Anregungen hat dann LEONE CAETANI zu seiner genialen, wenn auch umstrittenen Theorie des inaridimento ausgebaut ${ }^{2}$ ). Die arabische Wanderung ist nach dieser Lehre die letzte große semitische Wanderung, die sich aus der arabischen Halbinsel, der Völkerkammer der Semiten, über die Kulturländer ergoß. Neu ist nun die These,

s) Op. cit.

2) Annali dell' Islam II a. H. 12 §§ 105-II7. 
da $B$ diese Wanderungen - wie übrigens alle Wanderungen - durch einen sich über Jahrtausende hinziehenden Klimawechsel und die allmähliche Austrocknung des Landes hervorgerufen sind. Nicht religiöse Begeisterung, sondern der Hunger treibt die Araber über die Grenzen ihrer Halbinsel, wie er einst vor Jahrtausenden die semitischen Wanderungen des Altertums hervorgerufen hat.

Ich möchte mich voll und ganz zu dieser These bekennen; denn sie wird durch den historischen Tatbestand überreichlich begründet. Arabien ist seit Jahrhunderten vor Muhammed in Unruhe; Südaraber sitzen im Norden; die Grenzstämme drängen ins Kulturland; es hat wohl überhaupt nie Ruhe in Arabien gegeben, seitdem die ersten Völker über seine Grenzen getreten sind. Wir haben historisch beglaubigte Nachrichten von dem wirtschaftlichen Rückgang des Landes mit der ausdrücklichen Begründung des Wassermangels; Arabien ist, wie die alten Bauten und die klassischen Nachrichten beweisen, seit Jahrhunderten .im Rückgang. Man darf natürlich nicht annehmen, daß dies inaridimento in ein bis zwei Jahrtausenden erfolgt ist, sondern man muß Zehntausende von Jahren in Rechnung setzen; allerdings können durch unbekannte Ereignisse (Meeresströmungen, Abholzungen usw.) auch schnellere Klimaveränderungen sich vollzogen haben. Ferner reagieren die Völker auch nicht sofort auf solche Veränderungen. Die Araber säßen vielleicht heute noch innerhalb der Grenzen ihrer Halbinsel, wenn sie nicht den starken Rückhalt in der jungen Militärmacht des Islam gefunden und die Verhältnisse in Persien und Byzanz nicht geradezu zur Eroberung eingeladen hätten.

Der wichtigste Grund für diese These ist aber die Überlegung, daß einfach gar kein Motiv für diese kolossale Völkerbewegung übrig bleibt, als das wir t s c h a f $\mathrm{t} l \mathrm{i}$ c h e, nachdem einmal gezeigt wurde, $\mathrm{da} \beta$ das religiöse Moment nur eine sehr untergeordnete Rolle spielt. Wäre es den Arabern wirtschaftlich sehr gut ergangen, hätten sie in fest gegründetem Wohlstand gelebt, so hätte sie die Aussicht auf Beute nicht aus ihrer Heimat gescheucht. Ein gewaltiger religiöser Enthusiasmus hätte das wohl fertig gebracht, aber der würde sich dann außerhalb Arabiens anders betätigt haben als der junge Islam.

Die Triebkraft für die Ausbreitung der isla. mischen Herrschaftistalsodas wirtschaftliche Moment. Dies allein aber hätte das. Weltreich nie und nimmer begründet. Die arabischen Stämme waren in FluB; doch was bedeuteten sie in ihrer Isoliertheit gegenüber den großen Militärmächten an der Grenze? Die Stammeseifersucht machte aber jedes gemeinsame Vor- 
gehen unmöglich. In die s e m Punkte setzte der Islam ein; nicht als ob die Religion es fertig gebracht hätte, den alten Stammeshader in einem islamischen Brudertum aufgehen zu lassen - wissen wir doch, da $B$ der Gegensatz zwischen Süd- und Nordarabern erst im Islam seine sprichwörtliche Schärfe gewann -, aber als Muhammed zuerst weitere Stämme unter seine Fahnen zog, da überwog, wie wir das oben sahen, bereits längst der politis che Machtwille das religiöse Sentiment. Die Religion hatte ganz gewiß die politische Organisation von Medina erst ermöglicht; aus der Gemeinde erwuchs der Staat. Dann aber war es dieser $S t$ a a $t$, nicht die Gemeinde, der die arabische Völkerwanderung, die unabhängig von ihm entstanden war, für seine politischen Zwecke ausnutzte. Man sehe sich nur die Leute an, die das arabische Weltreich begründen! Ein Hăalid b. el-Walīd, ein 'Amr b. el-'As sind Herrenmenschen, Eroberernaturen, die sich um die Religion nicht kümmern, sie höchstens gelegentlich im macchiavellistischen Sinne ausnutzen. 'Abdallăh b. 'Omar und religiös beschauliche Naturen seines Schlages haben zur Ausbreitung des islamischen Reiches nicht das Mindeste beigetragen. Die medinensische Regierung mit ihren Generälen organisiert die Bewegung, doch wächst ihr diese bald über den Kopf. Es läßt sich mit Händen greifen, wie der Zentralregierung himmelangst wird vor ihren eigenen Erfolgen, wie sie es dann aber mit unleugbarem politischem Geschick fertig bringt, der Beutelust der Stämme ein Ziel zu setzen und eine organische Besetzung und Verwaltung der eroberten Gebiete allmählich in die Wege zu leiten ${ }^{\mathbf{x}}$.

Das einigende Schlagwort war wohl der Islam, aber im Sinne einer Weltherrsch aftder Araber. Dies nationale Bekenntnis war natürlich $\mathrm{reli}$ gi ös ge fä $\mathrm{rbt}$ wie alle großen Ideen orientalischer Völker, aber die begrifflich scheidende Kritik sieht den prinzipiellen Unterschied zwischen dem Nationalen und Religiösen in dieser Bewegung in der Stellung zur Bekehrungsfrage. Nun aber waren evtl. Bekehrungen von Nichtarabern nur möglich durch Angliederung an die arabischen Stammesverbände. Das nationale Moment überwog. Nicht Bekehrungs e if er, nicht glühende Worte eines begeisterten Propheten haben die Araber zu einer Welt$\mathrm{m}$ is sion mit Wort und Schwert hinausgetrieben, sondern die wirtschaftliche Notlage, die Unruhe der Stämme. Der überraschende $\mathrm{Erfolg}$. wurde herbeigeführt durch die glückliche Fügung e in es die Nation einenden Schlag wortes

r) Annali dell' Islam III passim. 
und durch den politis chen Machtwillen eines jungen von ehrgeizigen und gewaltigen Männern getragenen Staates.

So entsteht das große islamische Weltreich. Wie hat $\mathrm{sich}$ nun in ihm trotzderskizzierten Hemmungendie Islamisierung der unterworfenen Bevölkerung vollzogen?

$\mathrm{Da}$ das Schwert ausscheidet, möchte man zunächst an eine in te nsive Mission denken. Das ist auch die These des gründlichen, leider viel zu wenig bekannten Buches The Preaching of Islam von T. W. ARNOLD, der die Bekehrung hauptsächlich den unremitted labours of Muslim missionaries zuschreibt (S. 3). Er beklagt sich aber verschiedentlich, daß wir über diesen Prozeß selber so überaus wenig wissen. Der Mangel an Nachrichten ist durchaus nicht wunderbar. Der Islam kennt damals noch nicht das Gebot: „Gehet hin und lehret alle Welt! " Seine Ausbreitung vollzieht sich nicht als Selbstzweck, sondern als Konsequenz der Verbreitung seiner Bekenner. Die Mission des alten Islam wird sich kaum von der des modernen unterschieden haben, nur war sie mit weit größeren Schwierigkeiten verknüpft.

Ausbreitung der islamischen Religion hieß so viel, als Vermehrung der herrschenden Klasse und Verminderung der steuerzahlenden Untertanen. Das lag nicht im Interesse der Regierung und der arabischen Oberschicht. Es gab natürlich von Anfang an Idealisten, denen der Fiskus gleichgültig war, aber was hatten die zu bedeuten gegenüber der auf dem Gegensatz von Muslim zu Nichtmuslim aufgebauten Konstruktion des ganzen Staates?! Wie wenig die Araber anfangs mit Übertritten gerechnet haben resp. sie erstrebten, erhellt aus dem Umstand, daß sie die ganze wirtschaftliche Grundlage ihres Finanzsystems umändern mußten, als die Übertritte in größerer Menge begannen. Und doch trug der arabische Staat den Keim seines Unterganges in sich, sofern er nicht den Ubertritt zum Islam für Nichtaraber kurzerhand verbot; denn wenn ein Übergang aus der Klasse der Beherrschten in die Klasse der Herrscher durch die äußerliche Annahme eines anderen religiösen Bekenntnisses möglich war, so lag es von vornherein auf der Hand, daB diese soziale Trennung nicht lange bestehen würde. Ein Verbot des Übertritts widersprach aber der religiösen Tendenz des Islam, die niemandem den Glauben Muham. med's versagen konnte. Damit zerbrach der Gedanke der Universalreligiondas Nationalitätsprinzip des mit dieser Religion ursprünglich identischen States. Es zeugt für die Stärke des Staates und für den Mangel einer religiösen Propa- 
ganda, daB sich diese Konsequenz nicht gleich im ersten Jahrzehnt herausstelltc. Man kam crst hinter die den Staatsgedanken zersetzende Wirkung der Ausbreitung der Religion, als es zu spät war.

Gleich nach der Eroberung mögen einige Tausende den Islam angenommen haben; namentlich die in den Städten zusammenströmende Bevolkcrung wollte sich möglichst bald der Herrscherkaste assimilieren. Solange dic Millionen des flachen Landes unbekehrt blieben, konnte dieser Zuzug den Arabern für das Militärwesen, wie für das Wirtschaftsleben nur angenehm sein. Besonders dic oberen Zehntausend sahen, sofern sie nicht geflohen waren, sehr bald ihren Vorteil in einem möglichst engen $A$ nschluß an die Araber. In diesen Kreisen fanden wohl auch häufig Religionsdisputationen zwischen Muhammedanern und Christen statt, über die wir uns namentlich nach den Schriften des Johannes von Damaskus ein Bild machen können. Aber das trug doch wohl nur wenig zu einer wirklichen Verbreitung des Islam bei. Etwas stärker wirkte die Missionsarbeit, als sie von den religiös doch mehr oder weniger indifferenten Arabern auf die neu bekchrten Aramäer uberging, die in religiösen Dingen ganz anders ge. schult waren als die Araber. Auf diesen Punkt werden wir noch zurulkzukommen haben.

Viel stärker als dic Missionspredigt wirkte der wirts ch a f t li c he Vort cil, der dem Neubekehrten winkte, wenn er das arabische Burgerrecht als maulä crwarb. Er war damit von der schweren 'Tributsteucr befreit, auf deren Einzelheiten hier nicht eingegangen worden soll. Er wurde weiter der vielen Vorteile des städtischen Lebens teilhaftig und genoß endlich wenigstens einen Teil der sozialen Vorrechte des arabischen Elementes. Allzu schwer fiel ihm der Ubertritt nicht, da damals der Islam vor Ausbildung der Dogmatik sich gar nicht so schr vom Christentum unterschied. Er wirkte mehr wie eine neuc Selkte, als wic cine neue Religion und kam einer gewissen Reak. tion des semitischen Geistes gegen das durch und durch hellenisierte Christentum entgegen. Außerdem war es nun einmal die Religion der herrschenden Klasse, der Gott selber zum Sieg verholfen haben mußte. Unter dicsen Umständen waren die geistigen Widerstände gering, die sich dor Verwirklichung des materiellen Vorteils entgegensetzten. Und so konnte das wirtschaftliche Moment mit seiner ganzen Zugliraft ungestört wirken. Es spricht sehr für die Stärke der religiösen Bcharrlichkcit - ist aber viclleicht auch nur eine Folge des schlechten Nachrichtendienstes - da $B$ es immerhin uber ein halbes Jahrhundert daucrte, bis dic Übertritte zum Islam anfingen, das Stantsbudget aus $\mathrm{dem}$ Gleichgewicht zu bringen. Schon unter dem großen Staats. 
manne el-Hağgağ mußten Gegenmittel gegen diese unheilvollen Folgen der Ausbreitung des Islam gesucht werden. Die letzten Jahrzehnte des Omajjadenreiches sind überall erfüllt von Bestrebungen, dic zunehmende Islamisierung mit dem Staatsprinzip in Einklang zu bringen. Das führte zuletzt zu einer großen Reform der Steuergesetzgebung, von der wir erst die Umrisse kennen ${ }^{y}$ ). Danach bedeutete allerdings die Annahme des Islam nicht mehr Steuerfreiheit, aber doch wenigstens teilweise soziale Gleichstellung.

Die Vorbedingungen fur eine Islamisicrung großen Stiles waren aber erst gegeben, als $d$ a $s$ a $a$ b is che $S t$ a a $t$. prinzip überhaupt fiel, als der Zustand aufhörtc, daß die soziale Gliederung nach dem Nationalitätenprinzip statthattc. Dicse Veränderung vollzog sich im Laufe des ersten Jahrhunderts der 'Abba sidenherrschaft und hatte sehr mannigfache Gründe. Der wichtigste war dic auf die Dauer immer fühlbarer werdende Ü b c r le ge n. heit der materiellen und geistigen Kultur der unterworfenen Völker. Solange sich dic Araber auf ihre Heerlager konzentrierten, sich von der unterworfenen Bevölkerung fernhiclten und die Rolle von Grandseigneurs spiclten, die sich auf ihr Schwert stützen, vermochten sic ihre Supcriorität zu halten. Als aber immer neue Massen von Arabern aus Arabien nachströmten, als dic Kriege aufhörten, als der Araber nicht mehr Staatspensionär war, sondern sich sein Brot selbst zu verdienen hatte, als endlich seine Kaste durch das immer zahlreichere Eindringen von Maulás gesprengt wurde, da besaß er kein Gegengewicht mehr gegen die gewaltige Überlegenheit der unterworfenen Völker. Er paßte sich ihnen an, wo er sie erst nur hatte gewähren lassen, um schließlich abhängig von ihnen zu werden. Das war auf dem Lande ebenso, wic in der Stadt. Nach. dem der Araber erst einmal zum Landbesitzer, zum Baucrn, zum Kleinbürger geworden war, der wirtschaftlich abhängig wurde, oder doch zum mindesten dem gewandten Neubekehrten gleichgestellt war, da wurde es immer schwicriger, seine Prärogative zu bewahren.

Wie im Wirtschaftsleben ging es auf $\mathrm{g}$ cist $\mathrm{g} \mathrm{g} \mathrm{m} \mathrm{G}$ c b ic t. Die Aramäer, Griechen und Perser waren an Bildung und Tradition den Arabern so unendlich uberlegen, daß der Araber als der rohe tölpische Barbar angesehen wurde, der sich der feinen Sitte des vorderen Orients nur schwer anpaBt 2). Diesen Gedankengangen der anti.

2) Weclnausen, Das arabische Rrich, S. 297 月.; Beckex, Beilrdge eur Geschichle Ägyplens II, S. 81 A.; Papgri Schoth Reinhardt 39 II.

2) Vgl. Golozurk's Studien aber die jüubijie in Muhammedanische Studien $1,147 \mathrm{~A}$. 
arabischen Reaktion hatte der Araber bald keine tatsächliche Macht, keine gleichwertige Kultur oder Bildung, sondern eigentlich schließlich nur scine arabische Religion entgegenzusetzen, wodurch sie für ihn an Wort gewann und selbst der Gleichgaltige zu einer Betonung des Religiosen veranlabt wurde. Der religiose Nimbus war das cinzige Vorrecht, das ihm nicht genommen werden konntc.

Das Ubergewicht der Araber ware nicht so schnell den kulturellen Linnllussen erlegen, wenn die Staatsspitze das aristokratische Prinzip des arabischen Stantes energisch gewahrt halte. Aber auch dic Re. ficrung muBte sich, um zu wirken, den bestehenden staatsrechtlichen Anschauungen der Mehrzalıl der Bevölkerung anpassen, die übrigens dem Lihrgeiz des Individuums aber alle Maßen entgegenkam. Schon in dor Omajjiclenzeit sehen wir dic Anfänge, unter den 'Abbasiden aber ist die al torientalische Despotic mit der Vergött. lichung des Ilerrschers, dem Schutzwall des Zeremoniclls, dem Ausbeutungsinstrument der Bureaukratic und der Waffe der Sklavenginrden vollsthndig ausgebildet, oder richtiger gesagt wiederhergestellt. Damit wird dic Prillogative der Araber auch fur die Staatsspitze unertriglich. So gaugt der altorientalische Stat den aristokratischen Stant derAraberauf. Nun gibt es nicht melir cin Yerrschervolk uber den Unterworfenen, sondern lauter gleiche Knechte unter einem IIerrn. Damit ist die sozi a le Nivellierung vollrogen, die Knechte beeilen sich natürlich, dio Religion der IIorren anzunchmen, oder sic adoptieren sic durch Verselimelzung mit der fruheren Oberschicht. So breitet sich dic arabischo Sprnche aus und mit ihr dic Religion.

Jetat geht dio Bekehrung in schnellerem Tempo; den Arabern follte der missionarische Trieb gegenuber den Unterworfenen. Jetzt wurdo nber gar nicht melir von den $\Lambda$ rabern missioniert, sondern von Noubokehrten oder ihren Ableommlingen. Diese aber standen in ganz anderen Traditionen; sie kannten als frulhere Christen die Kirchlich. keit, und dio Geschichte ist voll Bewcisen für dic Rolle, dic unter den Aramtlorn die religiousen Frngen spielten. Stammten sie aber aus persischen Kreisen, so war ihnen der Begriff des Stantskirchentums und der Allgegenwart der Religion ebenso geliufig. Mit Wiederherstellung des despotischen Stantes der Chosroen erbluhte auch wieder der dem Arnber boi aller Betonung sciner religiösen Superioritit ganz fromdo Begrif des Stantsklerikalismusx). Jetzt lag

1) Vgl. Gocozuma, Ishmmisme at Fursisme (Actes du I. Congrds d'histoire des Religions) S. 12,16. 
die BekehrungderHeiden im Interesseder Staats. s pitze, während im arabischen Reich das Gegenteil der Fall war. Und der Herrscher fand Rückhalt in einer ganz neuen Klasse von Menschen, über die wenigstens die Anfänge der arabischen Zeit noch nicht verfügt hatten, die Theologen, die Juristen, die Dogmatiker, kurz die Schriftgelehrte $n$, um den mißverständlichen Terminus Klerikale zu vermeiden. Diese Leute konnten auf der Basis sozialer Gleichstellung von religiösem Ehrgeiz beseelt natürlich ganz anders wirken, als die arabischen Herren der Frühzeit gegenüber den unterworfenen Völkern, die sie nur ungern in ihren Kreis aufnahmen. In der 'Abbāsidenzeit wird es als Ruhmestat der Frommen erzählt, wenn Tausende in ihre Hände das islamische Glaubensbekenntnis abgelegt haben ${ }^{2}$ ). Solche Notizen beweisen, daß man die Bekehrung als verdienstlich ansah. Unter diesen Umständen ist es begreiflich, daB sich nun die Is la misierung sehr rasch vollzog und wohl schon im dritten und vierten islamischen Jahrhundert den Umfang erreichte, den noch der heutige Orient zeigt. In der späteren Zeit geht also die Ausbreitung des Islam zweifellos auf r e lig i ös e M o t i v e zurück, genau wie in der mekkanischen Zeit des Propheten. In der ganzen $Z$ wischenzeit wirkten aber wirt. schaftliche und politische $K r a ̈ f t e$, wenn auch nicht ausschließlich, so doch vielstärker als der Bekehrungse if er.

So nahm denn der moderne Orient allmählich den Islam als Religion an. Liegt nun in dieser Tatsache schon die Begründung für die religiöse Einheitszivilisation des Islam? Ganz gewiß nicht, das hieße Ursache und Wirkung verwechseln. Nicht die is lamische Religion hat die einheitliche Zivilisation erzeugt, sondern die aus ganz anderen Gründen entstandene einheitliche Zivilisation des Ka. lifenreiches ist die Voraussetzung für dic Aus. dehnung und das bis in die Gegenwart hinein siegreicheVordringender islamischen Religion.

Der wahre Zusammenhang wurde verdunkelt durch das Märchen von der sarabischen Kultur und ihrem Siegeslauf. Die Araber sollen die Zivilisation des Kalifenreiches geschaffen haben, und es gibt Leute, die den Arabern sogar die Mamlükenbauten oder die Alhambra aufs Aktivkonto schreiben. Das ist eine vollkommene Verkennung der Tatsachen. Es ist eine $\mathrm{philolog}$ is che Geschichtsbetrachtung,

3) ArNoLd, The Preaching of Islam S. 65. 
die ebenso schief ist wie die oben bekämpite theologis che. Weil die islamische Zeit mit der arabischen Wanderung begann, weil der vordere Orient seit den Kalifen arabisch spricht, weil seine älteren Literaturdenkmäler arabisch sind, deshalb soll auch die Zivilisation arabisch sein. Wie wenig davon wirklich arabisch sein $\mathrm{k}$ a n $\mathrm{n}$, ergibt sich schon aus dem Vorangehenden; wie wenig es tatsächlich is $t$, zeigt das Folgende.

Wo haben wir denn in der Kalifenkultur etwas t y $\mathrm{p}$ is c h Arab i s ch e s ? Im Staat? Gewiß in den Anfängen, aber wir haben eben gezeigt, daß die Araber nicht einmal bis in die Glanzzeit des Kalifenreiches die von ihnen geprägte Staatsform zu bewahren wußten. In der Verwaltung? Wer die Papyri kennt und wer die historischen Nachrichten unbefangen zwischen die juristischen Tendenztraditionen und die klassischen Nachrichten gestellt hat, der weiß, daß nicht die Weisheit der ersten Kalifen, sondern die byzantinische und persische Bureaukratie die "arabische" Verwaltung geschaffen haben. Wie in der Verwaltung, so war es in der ganzen materiellen Kultur und auch im Geistesleben. Trägt nicht selbst die arabische Religion in ihrem Lehrgebäude den Stempel der christlichen dogmatischen Kämpfe, der neuplatonischen und indischen Mystik? Ist die Naturwissenschaft und die Philosophie nicht von den Griechen; die Baukunst von den Byzantinern und Persern, die Geschichtswissenschaft von den Persern usw. usw.? Gewiß enthält die Zivilisation des islamischen Reiches einen starken arabischen Einschlag, so in der Poesie und namentlich auf gewissen Gebieten des Rechtes, wie Erbrecht, Eherecht und anderem, aber selbst ins Recht sind nur die alte Praxis der ein Jahrhundert lang herrschenden Oberschicht und die Vorschriften des Qorān's a u f g e $n$ o m m e n, ein großer Teil der Kasuistik stammt aus ganz anderen Quellen. Und ist es nicht überaus charakteristisch, daß von den "Wurzeln der Rechtswissenschaft" (ușül el-figh), die sich in Quellen und Prinzipien scheiden, nur die $Q$ u elle $n$ arabisch sind, die $P$ rin $z i p i$ e $n$ aber aus der von den Arabern vorgefundenen Zivilisation entnommen sind? Also von mehr als einem arabischen $\mathrm{E}$ in s chlag kann in der islamischen Zivilisation nicht die Rede sein. Das meiste, das uns als spezifisch arabisch anmutet, z. B. in den großen Adabund Hadittwerken, diesen unvergleichlichen Querschnitten durch die islamische Zivilisation, ist gar nicht arabisch, sondern vorderas iatis ch schlechthin, bestenfalls a $\mathrm{uch}$ arabisch, häufig aber durch und durch unarabisch. Wir werden nur getäuscht durch die literarische Form, die jegliche Maxime, wie jegliche Handlung des täglichen Lebens mit dem Vorbild des Propheten belegte. Die Araber 
haben sich einfach einer bereits vorgefundenen einheitlichen Zivili. sation angepaßt. Wie aber ist diese zustande gekommen?

Die Erklärung für die Möglichkeit einer is lamischen Einheitszivilisation liegt haupt. sächlich in der weltgeschichtlichen Tatsache des Hellenismus. So bizarr es klingt: Ohne Alexander den Großen keine islamische Zivilisation! Seitdem wir wissen, wie der Hellenismus in Indien, in Zentralasien gewirkt hat, haben wir erst den richtigen Maßstab gewonnen für die Stärke der Kulturmischung in Vorderasien während der Diadochenzeit. Ich wage nicht die Frage zu erörtern, ob schon die sogenannte »altorientalistische Kultur« ebenso universell war wie die islamische Zivilisation $\mathrm{I}$ ), die starke Einheitlichkeit der vorderasiatischen Zivilisation ist jedenfalls nach der Diadochenzeit mit Händen zu greifen. Zwar schafft die Trennung in zwei Reiche, das römische und das parthische, wie später das byzantinische und das sassanidische, eine neue große Kluft, und es ist selbstverständlich, daß der römische Orient eine starke abendländische Tünche empfing, während im Osten die hellenistischen Kulturelemente immer mehr im Asiatismus untergingen. Aber vom dritten Jahrhundert unserer Zeitrechnung ab, ja schon früher, läßt sich beobachten, wie auch der römische Orient sich immer mehr as iatisiert, und wie schlieBlich das byzantinische Reich sich dem sassanidischen immer mehr anpaßt. Wir stehen hier der großen Bewegung gegenüber, mit der Asien auf den Hellenismus und das römische Weltreich reagierte.

Die Grenzen zwischen Ost und West wurden immer labiler, ja selbst der politische Rahmen zerrèißt schließlich in den ständigen Kämpfen zwischen Persien und Byzanz, und im kleineren Maßstabe mögen die Züge Hosrau's II. und des Heraklius eine ähnliche Bedeutung gehabt haben wie die Züge Alexanders des Großen, wenigstens im Hinblick auf Herstellung einer einheitlichen, wenn auch gemischten Zivilisation.

Das bunte Kulturgemisch des vorderen Orients fand seinen vollendetsten Ausdruck in der a ramäischen Christenheit ${ }^{2}$ ). Schon die Sprache dieser Leute zeigt nicht nur in ihrem Wortschatz, sondern in ihrer ganzen Diktion eine seltsame Mischung von semitischen, griechischen und persischen Elementen. Auch bildeten sic durch ihre kirchliche Gemeinschaft eine wichtige Brücke, auf der Kulturgüter mühelos die Kluft der Reichsgrenzen überschritten. Vor

1) These von Huco Wisckure.

2) Vgl. dazu mein Christentum und Islam, S. 18 ff. Ähnliches gilt naturlich von der koptischen und nordafrikanischen Christenheit, doch aberwiegt der EinfuB der Aramaer. 
allem aber stellten sic trotz unzähliger fremder Einschläge und Rassenmischung eine gerade durch die Mischung entstandene ethnische Einheit dar, die auf äußere Einflusse ähnlich reagierte. Es ist bekannt, daB dic arabische Eroberung erst dort ernstliche Schwierigkeiten fand, wo sie nationalen bodenständigen Kräften gegenübertrat wie in Persien und in Nordafrika. Hier fuhre ich diese Tatsache nur zur Illustration der kulturellen Einheit des aramäischen Sprachgebietes an. Scit FraENKEL'S und NOLDEkE's Studien ${ }^{3}$ ) wissen wir, wie abhängig die Araber schon in vorislamischer Zeit von der aramäischen Zivilisation gewesen sind. Sie haben nach der Eroberung Vorderasiens nicht anders gehandelt als vorher, sondern einfach die aramäische Zivilisation in extenso ubernommen, ja einfach weiterge le bt. Von gewissen rein arabischen Einschlägen ist schon die Rede gewesen.

Nun bekäme man aber doch ein sehr $\mathrm{s} c \mathrm{~h}$ i e f es Bild von der islamischen Zivilisation, we n $\mathrm{man}$ sie einfach der ara. mäischen plus arabischem Einschlag gleichsetzen wollte. Das ist für die allerersten Anfänge richtig, und die wichtigste Basis für die islamische Einheitszivilisation bleibt die aramäische unter allen Umständen. Aber nur die Basis. $D$ ie is lamische $Z$ ivilisation ist eine Weiterentwi cklung deraramä is chen und zwar in der gleichen Richtung, in der sich die aramäische Zivilisation bis zum siebenten Jahrhundert entwickelt hatte.

Die Entwicklungstendenz des vorderen Orients in den letzten Jahrhunderten vor Muhammed war, wie schon angedeutet, die allmählich immer stärkere Asiatisierung. Das Schwergewicht und die Quellen der herrschenden Zivilisation hatten sich vom Mittelmeer immer mehr nach dem innern Asien verschoben. $D$ i e se $r$ ProzeBsetztsich nun im islamischen Reiche anda uernd fort. Die Erklärung dafür liegt auf der Hand. Sie liegt $n \mathrm{ich}$ tinder Religion des Islam, sie liegt in der poli$\mathrm{t}$ is chen $E$ inheit des vorderen Orients, in der weltgeschichtlichen Tatsache des vorderasiatischen $E$ in he its $\mathrm{t}$ a a tes. Bisher hatte sich eine kulturelle Einheitlichkeit entwickelt, t rot $z$ der staatlichen Trennung, trotzdem der vordere Orient politisch nach dem Westen gravitierte. Nun wurden plötzlich weite Provinzen mit dem persischen Reiche zu einer Einheit verbunden; dadurch wurden ihnen die wichtigsten $Z$ uströmungen der abendländischen Antike unterbunden, während alle Schranken gegenüber den asiatischen Einflüssen fielen.

3) Fraenker, Die aramäischen Fremdwörler im Arabischen. 
Dieser historische Zusammenhang war natürlich weder den Arabern noch den Aramäern klar. So konnte sich sogar während der ganzen Omajjadenzeit die Reichsresidenz ganz an der Peripherie erhalten. Das Ubbergewicht von Damaskus resultierte aus dem zufälligen Gang der Ereignisse und aus dem anfänglichen Übergewicht des arabischen Elementes. Aber schon während der politischen Vorherrschaft von Damaskus verrät sich immer deutlicher das wirtschaftliche und kulturelle Übergewicht des 'Irāq. Die Verlegung der Residenz nach Bagdad ist dann der deutlichste Beweis für die Unvermeidlichkeit der angedeuteten Entwicklung. Von da ab ist das Kalifenreich nichts anderes, als die Fortsetzung des Chosroenreiches auf breiterer politischer Basis. So ist es denn ganz natürlich, daß die Kalifenkultur immer stärkere persische Züge zeigt, und daß sie im Fortgang der Geschichte noch weiter von Zentralasien her, vom Türkentum, ja von China beeinflußt wird. Die Kalif e $\mathrm{kultur}$ ist alsonicht arabisch, aberauchnichtrein a ra. mäisch oder persisch, aber trotzdem eine geschlossene Einheit a f der Basiseines Einheits sta a tes. Da dieser Staat der islamische Staat war, so spricht man mit vollem Recht von einer is $\mathrm{l}$ a $\mathrm{m}$ is $\mathrm{ch}$ e $\mathrm{Z} \mathrm{Z}$ ivilis a $\mathrm{t}$ i o $\mathrm{n}$.

- Verweilen wir noch einen Augenblick bei den kulturellen Folgen des Einheitsstaztes, um uns diesen Prozeß recht klarzumachen. In früheren Arbeiten habe ich oft betont, wie sehr unter. den Arabern alles beim alten bleibt. In unserem jetzigen Zusammenhange muß mindestens ebenso stark betont werden, daß sich trotz lokaler Selbständigkeit durch die Tatsache der einheitlichen politischen Leitung eine immer größere Mischung vollzieht. Das geschieht zunächst auf dem Gebiete des Wirtschafts lebens. Die alten Zollgrenzen bestehen zwar fort, aber der Handel, jener größte Kulturträger überhaupt, vermag doch nun ganz andere Gebiete gleichmäßig zu durchdringen, die alten Umschlagsplätze an den früheren Grenzen werden Durchgangsstationen; man geht an dem Zollerheber vorbei, wie es unendlich oft in der Tradition heißt (marra bi-l-säsir). Und genau wie der Warenhandel wirken dann mit Entwicklung der kapitalistisch gefärbten Unternehmungen die Riesenspekulationen und die Trans. aktionen der GroBunternehmer, die in der Blütezeit des Kalifats das ganze Reich überspannen. Vor allem aber entwickelt der Staat selber durch Ausdehnung der lokalen Le it u r g i e auf das gesamte Reich ein Hinüber- und Herüberfluten der kulturellen Anregungen, wie es vor der Herstellung des Kalifenreiches einfach undenkbar war. Ich hatte beabsichtigt, diesen ProzeB hier des Näheren zu skizzieren, kann 
aber jetzt auf die Darstellung von ERNST HERZFELD in diesem Hefte verweisen $\mathrm{z}$ ), der ich mich voll und ganz anschließe. HERZFELD zeigt an dem Musterbeispiele der Entstehung der islamischen Kunst, wie gewaltig die wirtschaftlichen Kräfte die Entstehung der islamischen Einheitszivilisation gefördert haben.

Und genau ebenso war es auf geistigem Gebiet. Hier beginnt der Austausch allerdings erst, nachdem die wirtschaftlichen und politischen Kräfte gewirkt haben, dann aber wird die neue $\mathrm{E}$ i n heitsreligion zu einem festen Bindemittel zwischen den in dem neuen Staate zusammengefaßten früher selbständigen Bevölkerungselementen. Die religiösen Scheidewände der Vergangenheit waren ebenso gefallen wie die politischen. Nachdem einmal die islamische Religion in der oben skizzierten Weise zu einer Macht geworden war und das gesamte geistige Erbteil der früheren Religionen sich assimiliert hatte, da wurde das "Suchen der Tradition" (țalab el-hadīt ), wie GoLDZIHER uns gezeigt hat, zu einem unendlich wichtigen Faktor in der Herstellung einer nun islamisierten geistigen Einheit. Vom Atlantischen Ozean bis nach China hinein entstand eine einheitliche religiöse Wissenschaft, die von den Schriftgelehrten assimilierten Lehren eines Aristoteles wanderten mit anderen geistigen Gütern durch das ganze Reich, ja dann über seine Grenzen hinaus bis in das Lop-Nōr oder an den Tschadsee.

Eine besonders wichtige Folge des Einheitsstaates war nun auch, $\mathrm{da} B$ die Außengebiete $d$. h. die ursprünglich nicht zur aramäischen Zivilisation gehörigen Länderkomplexe wie Persien, Ägypten, Afrika ihren Teil zu der neuen Einheit beisteuern konnten. Auch als diese Länder sich früh wieder von dem Reichsganzen trennten, blieb doch immer eine ideelle Einheit und ein tatsächlicher Austausch bestehen, wenn auch diese Gebiete, wie z. B. Spanien und Iran, eine starke Eigenart zur Schau tragen.

So wird allmählich aus dem aramäischen See durch starke Zuflüsse von mannigfachen Seiten das große einheitliche Meer der islamischen Zivilisation. .. Und jetzt beginnt a uch die religiöse Durchsetzung, die im Laufe der Entwicklung zum Charakteristikum dieser neuen Einheit wird. Ich brauche nicht zu wiederholen, was ich oben über die Ausbreitung der islamischen Religion gesagt habe. Die Predigt Muhammed's ist nicht die alleinige Ursache dieser Klerikalisierung, sie verbindet sich mit viel älteren Tendenzen, mit dem christlichen Klerikalismus und dem persischen Staatskirchentum.

x) Siehe unten S. 60 ff. 
Der Sieg des Kalifats (ḩilāfa) der 'Abbāsiden über das Königtum (mulk) der Omajjaden ist nicht nur der Sieg der religiösen Idee Muhammed's über die heidnischen Tendenzen der mekkanischen Aristokratie, sondern zugleich der Sieg des persischen und christlichen Staats. kirchentums, der ultrareligiösen Weltauffassung des alten Orients überhaupt über die religiöse Indifferenz des rein weltlichen Reiches der arabischen Nation. Der Name Islam bleibt, aber sein Inhalt wird ein vollkommen anderer.

Jetzterstist der Islam die Mischung von Religion, Staatsideal und Zivilisation, die ihn zu einer Weltmission befähigt. Wenn auch in seiner späteren Zeit auf beschränkten Gebieten national begründete Landeskirchen entstehen wie die persische Schr̃a, wenn auch Riten und Sekten sich absondern und separatistische oder atavistische Gelüste sich in der Form von Ordensbildungen betätigen, wenn auch ferne Außengebiete, wie der chinesische Islam, dem erdrückenden Übergewicht der sie umgebenden Nationalkultur nicht $\mathrm{zu}$ widerstehen vermögen, die $\mathrm{T}$ a t s a che e in er groBen is la $\mathrm{m}$ is chen $\mathrm{E}$ in heit bleibt nichtsdestoweniger bestehen, ja sie wird dem aufkommenden Europa gegenüber immer mehr betont. Diese Einheit liegt greifbar vor uns in dem gewaltigen Lebensreglement der Scharĩa.

Interessant ist nun zu sehen, wie die Religion, die sich auf Grund all der geschilderten Faktoren ausgebreitet hat, bestehen bleibt, ja erstarkt, als die Momente, die sie emporgebracht haben, verblassen und untergehen. Aus dem Einheitśstaat wird das I d e a 1 des Einheitsstaates. Man meint meistens, dies Ideal wäre den wirklichen oder vorausgesetzten Zuständen der goldenen Zeit der orthodoxen Kalifen entnommen. Ich glaube nicht. Der ungeheuren Tatsache des Omajjaden- und 'Abbāsidenkalifats sind die Züge entlehnt, die man dann auf die orthodoxen Kalifen übertrug, wobei gern zugegeben sei, daß diese Lehren sich in der Opposition gegen die Omajjaden entwickelt haben, dann aber unter der Sanktion der 'Abbāsiden, die gerade an das Regime der orthodoxen Kalifen anknüpften, ihre Ausgestaltung erfahren haben. Das Ideal des Einheitsstaates zwingt die späteren Sultane zur Beibehaltung der Investitur durch den Schattenkalifen. In der Frühzeit des Islam stand die religiöse Idee im Dienste des Machtgedankens, unter den 'Abbāsiden hatte man alles getan im Interesse der Macht, den religiösen Staatsgedanken zu entwickeln und zu verbreiten; die Sultane müssen dann schon mit ihm rechnen. Dann aber verblaBte er immer mehr, doch nennen sich noch heute islamische Machthaber gern Kalifen. 
Wichtiger noch als das Staatsideal ist das Lebens- und Bildungsideal des Muhammedaners, das sich uns im islamischen Rechte darstellt und aufs engste mit der Religion verbunden ist. Es ist die kanonisierte und theoretisierte Praxis jener Blütezeit, die vortrefflich in das Milieu paßte, in der sie schon vor dem Islam bestand. Als aber nun in späteren Jahrhunderten der Islam ohne staatlichen Rückhalt rein als Religion und Zivilisation meist auf dem Wege des Handels uber die Grenzen des alten Kalifenreiches hinausdrang, da verhielt sich das Lebensideal zu der Praxis jener neuen Länder wie das Ideal des Staates zur traurigen Wirklichkeit. So entstand der bekannte ${ }^{\mathrm{y}}$ ) Gegensatz zwischen religiöser Forderung und volkstümlicher Tradition, zwischen šarz' $a$ und 'äda. War die Scharīa schon dort, wo sie entstand, mehr ein Ideal als eine Tatsache, so ist sie auf fremdem Boden ein reines Ideal geworden. Eine wirkliche Anpassung, ein wirkliches Leben war nur den rein religiösen Teilen der Schari'a beschieden. So ist denn schließlich in der Gegenwart das religiöse Moment des Islam zu dem eigentlich wirksamen geworden und die tatsächliche Verbindung der Muhammedaner aller Zungen und Zonen beruht heute in der Ge meinsamkeit des Bekenntnisses, in der Einheit i hrer Idè a le. Die erstaunliche Ausbreitung des Islam in primitiven Ländern erfolgt aber auch heute noch dank der Mischung von Religion, Kulturträger und staatenbildender Kraft, als die sich der Begriff Islam darstellt. Das einende Band ist die Religion, an der erst die nationalen und liberalen Bestrebungen der Gegenwart zu rütteln beginnen.

Der Islam ist also kein so einfaches Gebilde, wie man ihn häufig hinstellt. Mit geographischen Schlagworten von dem Islam als Kind der Vegetationsform der Steppenländer kann man wohl Laien verblüffen ${ }^{2}$ ), man beweist aber damit nur, daß man das Wesen dieser großen historischen Erscheinung nicht begriffen hat. Auch wer den Islam unter den Begriff der "Kultur der Araber" bringen will, steht einem wirklich geschichtlichen Verständnis des hier liegenden Problems doch noch recht fern. Wem endlich zur Erklärung der gegenwärtigen Tatsache des Islam der Qorān und das Leben Muhammed's genügen, dem ist überhaupt nicht zu helfen. Da solche Gedanken aber bis in die letzte Zeit hinein immer und immer wieder der breitesten Öffentlichkeit vorgesetzt werden, war es wohl nicht ganz zwecklos, diese

3) Vgl. die zahlreichen Arbeiten von SNouck Hurgronje.

2) E. BAnSE, Die Atlasländer (Aus Natur- und Geisteswelt Bd. 277) S. 26 u. passim. 
Zeitschrift mit dem Versuch zu eröffnen, die großen Linien der islamischen Entwicklung zu zeichnen.

So viel Subjektives und Hypothetisches auch in dieser Skizze liegt, in den Hauptsachen fußt sie auf gesicherten Vorarbeiten. Möge diese Zeitschrift dazu beitragen, das skizzierte Bild, wenn nötig, umzugestalten resp. zu modifizieren, oder aber, wenn es richtig ist, zu vertiefen und $\mathrm{zu}$ detaillieren.

Quod felix faustumque sit. 